\title{
Metamodel assisted design optimization of piezoelectric flex transducer for maximal bio-kinetic energy conversion
}

\author{
Liheng Luo ${ }^{1}$, Dianzi Liu ${ }^{2}$, Meiling Zhu ${ }^{3}$ and Jianqiao $\mathrm{Ye}^{1}$
}

\begin{abstract}
Energy Harvesting Devices (EHD) have been widely used to generate electrical power from the bio-kinetic energy of human body movement. A novel Piezoelectric Flex Transducer (PFT) based on the Cymbal device has been proposed by Daniels et al. (2013) for the purpose of energy harvesting. To further improve the efficiency of the device, optimal design of the PFT for maximum output power subject to stress and displacement constraints is carried out in this paper. Sequential Quadratic Programming (SQP) on metamodels generated with Genetic Programming from a 140-point optimal Latin hypercube design of experiments is used in the optimization.
\end{abstract} Finally, the optimal design is validated by finite element simulations. The simulations show that the magnitude of the electrical power generated from this optimal PFT harvesting device can be up to $6.5 \mathrm{mw}$ when a safety design factor of 2.0 is applied.

\footnotetext{
${ }^{1}$ Department of Engineering, Lancaster University, Lancaster, UK

${ }^{2}$ Faculty of Science, University of East Anglia, Norwich, UK

${ }^{3}$ College of Engineering, Mathematics and Physical Sciences, University of Exeter, Exeter, UK Corresponding authors:

Dianzi Liu, Faculty of Science, University of East Anglia, Norwich, UK

Email: dianzi.liu@uea.ac.uk

Jianqiao Ye, Department of Engineering, Lancaster University, Lancaster, LA1 4YR, UK

Email: j.ye2@lancaster.ac.uk
} 


\section{Keywords}

Energy harvesting, piezoelectric, parametric optimization, design of experiments, metamodel

\section{Introduction}

In recent years, the rapid development of low power consuming devices, such as aircraft structural health monitoring (Sohn, et al., 2002) and portable communication devices (Olodort R and Cazalet P, 2004), have resulted in high demands for mobile energy harvesters, whose primary function is to reduce the use of battery. Consequently, the energy conversion efficiency of energy harvesters has become a challenging topic for researchers because the low power output of the mobile energy harvesters cannot satisfy the high power requirement of the devices.

There are many energy resources that can be harvested from the ambient environment. According to Harb (2011), micro-energy, which is produced on a small-scale from a low carbon source, can be mechanical, electromagnetic, thermal, electrical, solar or biological energy. Various micro energy harvesters have been designed to harvest energy from ambient environment and to power mobile devices, such as the wearable thermoelectric generator(TEG) (Krishna et al., 2011) and the cantilevered bimorphs piezoelectric vibration harvester (Khalid et al., 2015). The development 
and applications of micro-scale energy harvesters, including thermoelectric, thermo-photovoltaic, piezoelectric, and microbial fuel cell energy harvesters, have been reviewed by Krishna and Mohamed (2014). Piezoelectric energy harvesting has received great attentions since piezoelectric materials have good electrical-mechanical coupling effects. There have been a number of reviews that are specifically on piezoelectric energy harvesters and piezoelectric materials (Heung et al., 2011; Li et al., 2014; Siddique et al. 2015), which evidenced the recent and rapid development of this special form of energy harvesters.

To improve the efficiency of mobile piezoelectric harvesting devices, design optimization has been carried out to maximize the power output. Reinhard (1990) developed finite element equations for modelling piezoelectric structures and Silva and Kikuchi (1999) applied topology optimization method (TOM) to obtain the efficient layout of piezoelectric materials. They found an optimal distribution of the materials and void phases using finite element model and then generated the optimal design of the piezoelectric transducer. Kögl and Silva (2005) proposed a new material model that was based on the SIMP (solid isotropic material with penalization) to improve the design of piezoelectric actuators using topology optimization. Anton and Sodano (2006) concluded in a review that the efficiency of a piezoelectric power generator could be improved by better piezoelectric configurations, circuit or method of energy storage. Lu et al. 
(2011) proposed a maximum power point tracking scheme based on a time-multiplexing mechanism for general piezoelectric energy harvesters to improve the efficiency of energy harvesting from the circuit. Deng et al. (2015) maximized the output of a piezoelectric vibration energy harvester by finding the optimal damping ratio and electromechanical coefficient. Using TOM and SIMP, Kiyono et al. (2016) successfully improved the performance of piezoelectric energy harvesters. Zhou et al. (2016) proposed a flexible longitudinal zigzag structure (FLZS) to improve the low frequency piezoelectric vibration energy harvesting. Zhao et al. (2016) employed the synchronized charged extraction circuit to enhance the galloping piezoelectric energy harvesting.

In this paper, the novel piezoelectric energy harvester, Piezoelectric Flex Transducer (PFT) that was developed base on the Cymbal device (Newnham et al., 2000), is optimized to achieve the maximal bio-kinetic energy conversion. First, an introduction of a coupled piezoelectric-circuit finite element model (CPC-FEM) representing the Cymbal energy harvesting device is given and the model is validated against published results. Based on the developed CPC-FEM model, the response functions are calculated and then replaced by metamodels, which are constructed using Genetic Programming from a 140-point optimal Latin hypercube design of experiments. Finally, parametric optimization of the PFT is carried out using Sequential Quadratic Programming (SQP) 
on the metamodels to obtain the optimal design. The finite element simulation of the final optimal design shows that the magnitude of the electrical power by this PFT harvesting device can be up to $6.5 \mathrm{mw}$ when a safety design factor of 2.0 is applied.

\section{Development of the coupled piezoelectric-circuit finite element model and its validation}

The Cymbal device was originally designed as a piezoelectric actuator. The structure, function and applications of the Cymbal device were reviewed by Newnham et al. (2000). The cymbal device consists of two endcaps and a piezoelectric disk, which can increase power output by up to 40 times compared to the use of only a piezoelectric disk alone (Kim et al. 2007). However, the traditional Cymbal transducer was unable to stand a force of more than $50 \mathrm{~N}$, which means that it cannot harvest the bio-kinetic energy from, e.g., foot fall of human. In order to improve the Cymbal device for the purpose of bio-kinetic energy harvesting, Daniels et al. (2013) developed the Piezoelectric Flex Transducer (PFT) using a coupled piezoelectric-circuit finite element model (CPC-FEM) in ANSYS (2010). The CPC-FEM model was validated by comparing the simulation results of the Cymbal device with the ones from experiments.

Based on the research by Daniels et al. (2013), the CPC-FEM model is further developed for the optimal design of the PFT in this paper. The PFT was composed of a top endcap, a bottom endcap, 
two substrate layers and a piezoelectric plate. The PFT was made into a rectangular shape to retrofit into a shoe and tested subject to a force greater than $1 \mathrm{kN}$. This configuration of the PFT device can efficiently transfer the force from the endcaps to the piezoelectric disk. In order to increase the strength of the joint part, the area of the vulnerable adhesive interface between the endcaps and the piezo disk has been enlarged and substrate layers are also added. The geometric parameters of the PFT are shown in Figure 1(a) and (b). The detailed FE model of the PFT shown in Figure 2 is used to perform the simulations and structural optimization for maximal electrical power output. In the FE model, SOLID226 element was selected for modelling the PZT plate. This element is a 3-D couple field hexahedron of 20 nodes and suitable for the analysis of piezoelectric structural responses. SOLID95 element was selected for endcaps, which is also a hexahedral element type with 20 nodes. CIRCU94 element was used for the resistor connected between the positive and negative electrodes. The adhesive layers between the metal endcaps and the piezoelectric material are ignored in the finite element model since they are very thin. The geometric and material parameters are the same as those chosen in Daniels (2014). 


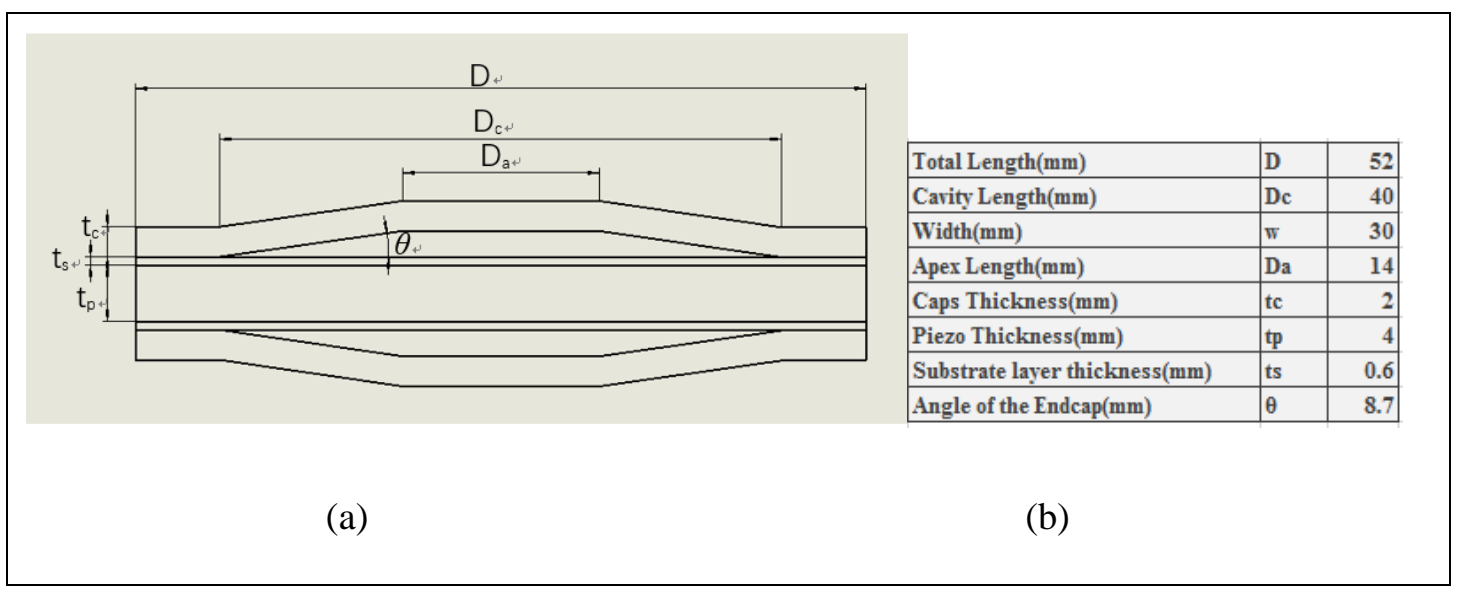

Figure 1. (a) Cross section view of PFT (b) Value of geometric parameters of PFT.

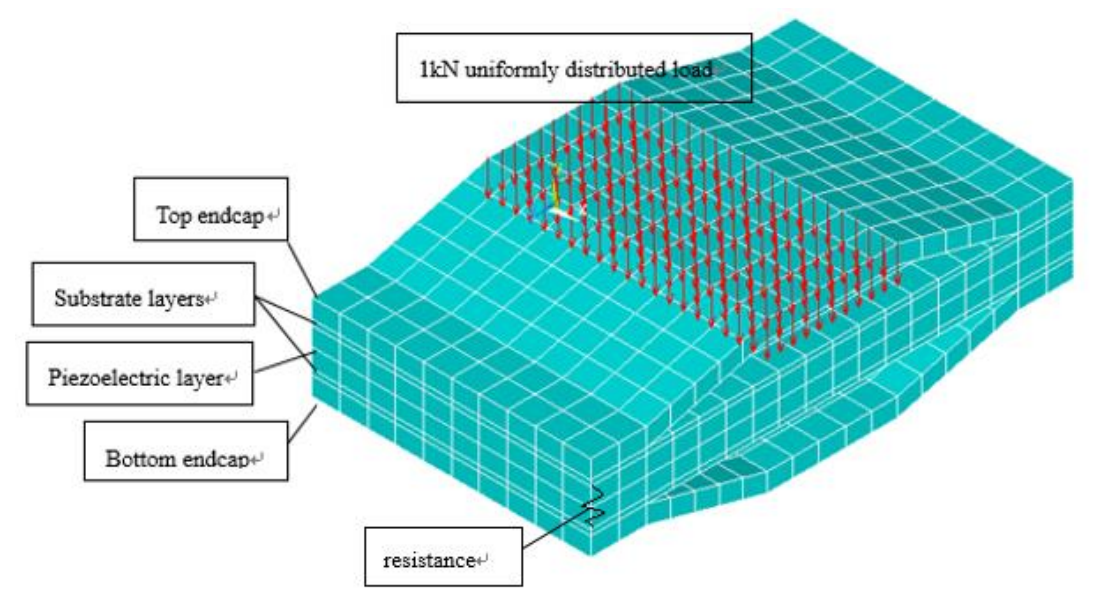

Figure 2. FEM of piezoelectric flex transducer.

In general, the accuracy of the predictions using the above CPC-FEM model depends on the size of the elements used. A discussion on how the meshing density affects the simulation results will be presented in the Convergence analysis of the developed PFT Section. To validate the model developed in this paper, a comparison with the results by Daniels (2014) is shown in Figure 3 for 
the PFT subjected to $1 \mathrm{kN}$ uniformly distributed load (UDL) downward from the apex with a frequency of $2 \mathrm{~Hz}$, where a resistor connecting the top and the bottom surface of the piezoelectric layers as the respective positive and negative electrodes. A resistant load ranging from $0.5 \mathrm{M} \Omega$ to $10 \mathrm{M} \Omega$ is used to study its effect on the electrical power output.

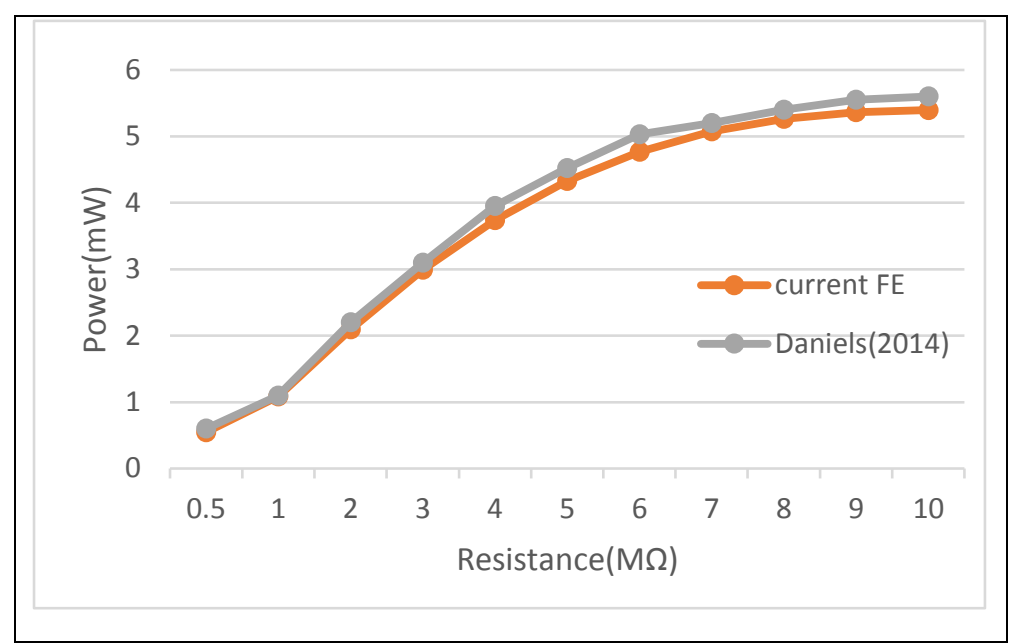

Figure 3. Comparison of the results from the current model and Daniels (2014).

A good agreement between the results from the converged CPC-FEM model and the ones obtained in Daniels (2014) is shown in Figure 3. Therefore, the converged CPC-FEM model will be used as the virtual experimental tool in the following metamodel based optimization process to maximize the power generation capacity of the original PFT design.

\section{Parametric Optimization of the Piezoelectric Flex Transducer (PFT) Device}


To determine the geometric parameters of the PFT device for the best design configuration in terms of the maximal power generation subject to stress and displacement requirements, parametric optimization of the PFT device is performed in this section. The design for the PFT structure is a multi-parameter optimisation problem, for which a metamodel-based optimization technique is used to obtain the optimal solution describing the PFT geometry. The methodology for performing parametric optimization consists of five steps and they are:

\section{Design of Experiments (DOE)}

II. Metamodel building by Genetic Programming (GP)

III. Finite element analysis of the PFT

IV. Design Variables, Constraints, and Loads for Parametric Optimization

V. Optimal design of the PFT by Sequential Quadratic Programming (SQP)

These five steps are integrated to perform the multi-parameter optimization of the PFT device design and explained in the following sub-sections. In this paper, parametric optimization is applied to obtain the detailed PFT design using Sequential Quadratic Programming on the global metamodels generated with Genetic Programming from a 140-point optimal Latin hypercube 
Design of Experiments, each of which represents a specific PFT design defined by a set of parameters input.

\section{Design of Experiments (DOE)}

Design of Experiments (DOE) is a powerful statistical technique (Box, 1980) to study the effect of multiple variables simultaneously. The DOE can economically satisfy the needs of problem solving and successfully deal with design optimization projects in various engineering subjects. By applying this technique, the time required for experimental investigations or computer experiments can be significantly reduced. Therefore, an approximation model for the response of the interest built by DOE design points is utilized to solve complex optimization problems to reduce computational cost, where a design point is related to a specific set of design parameters. However, the quality of a metamodel strongly depends on an appropriate choice of the Design of Experiments (DOE) type and the sampling size (Forrester et al., 2008). To improve the quality and efficiency of DOE, a uniform Latin hypercube DOE based on the use of the Audze-Eglais optimality criterion (Audze and Eglais, 1977), is used in this paper. The main principles in this approach are as follows: 
- The number of the set of design variables (same for each design variable) is equal to the number of experiments and for each set of the design variables, there is only one experiment allowed;

- The points corresponding to the experiments are distributed as uniformly as possible in the domain of design space where each design parameter is defined as an independent coordinate in the design space. Thus the distance between two neighboring points, e.g., points $\mathrm{p}$ and $\mathrm{q}$ representing two different sets of designs, can be calculated as Euclidean distance using Pythagorean formula. There is a physical analogy of the Audze-Eglais optimality criterion with the minimum of potential energy of repulsive forces for a set of points of unit mass, if the magnitude of these repulsive forces is inversely proportional to the squared distance between the points.

As a result, one has

$$
U=\sum_{p=1}^{P} \sum_{q=p+1}^{P} \frac{1}{L_{p q}^{2}} \rightarrow \min
$$


where, $P$ is the total number of points, $L p q$ is the distance between points $p$ and $q(p \neq q)$. Minimizing $U$ produces a system (DOE) where points are distributed as uniformly as possible in the design space.

Following this extended optimal Latin hypercube design of numerical experiments (DOE), the FE analyses of the proposed DOE are performed at the 140 uniformly distributed design points to optimise the PFT. The bar chart of the minimum distances between the sampling points is shown in Figure 4, which indicates a good uniformity of the DOE.

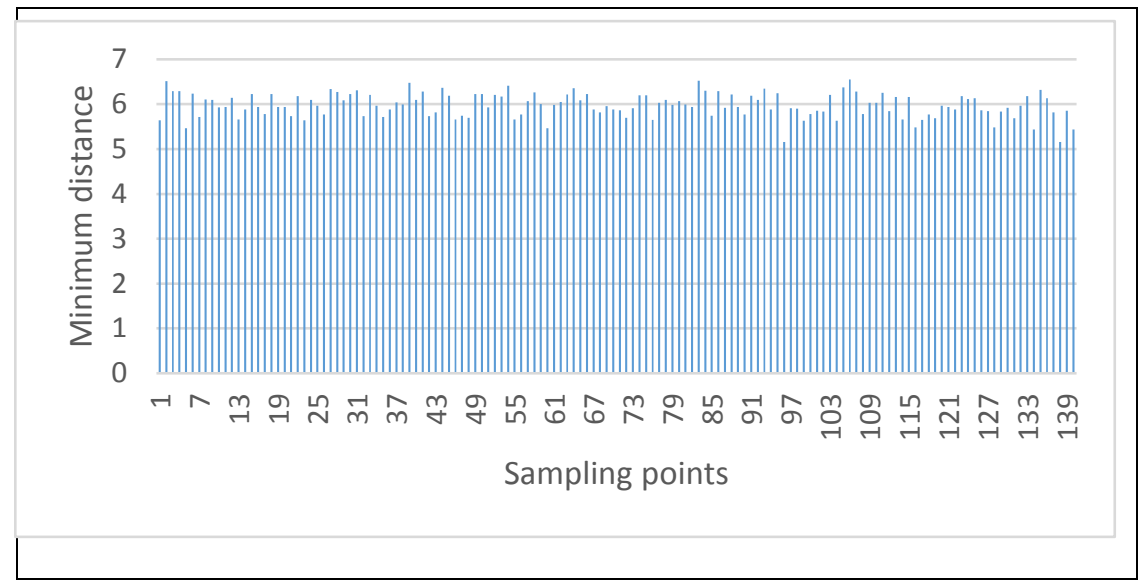

Figure 4. Minimum distances between points in 140-point optimal Latin hypercube design of experiments.

Metamodel building by Genetic Programming (GP) 
Genetic programming methodology (GP) (Armani et al, 2011; Koza, 1992) is a systematic way of selecting a structure of high quality global approximations. Selection of the structure of an analytical approximation function is a problem of empirical model building. Selection of individual regression components in a model results in solving a combinatorial optimization problem. Even if a bank of all regressors is established (which is a difficult problem on its own), the search through all possible combinations would result in prohibitive computational effort. GP is based on the same basic methodology as genetic algorithms (GA). While a GA uses a string of numbers to represent the solution, GP creates a population of computer programs of a certain structure. In this case of design optimization, the program represents an empirical model to be used for approximation of a response function. For example, a tree structure-based typical program, representing the expression $\left(\mathrm{x}_{1} / \mathrm{x}_{2}+\mathrm{x}_{3}\right)^{2}$, is shown in Figure 5.

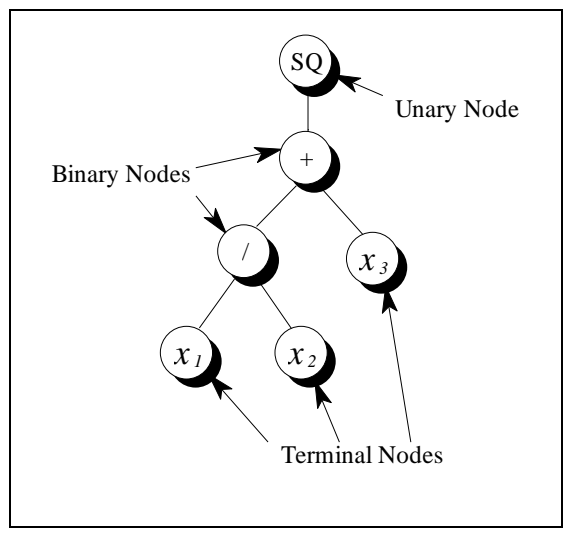

Figure 5. Typical tree structure for $\left(\frac{x_{1}}{x_{2}}+x_{3}\right)^{2}$. 
The genetic programming code was first developed according to the guidelines provided by Koza (1992), then implemented for symbolic regression tasks by Armani et al. (2011). The common genetic operations used in genetic programming are reproduction, mutation and crossover, which are performed on mathematical expressions (metamodels) stripped of their corresponding numerical terminals. Parameters are inserted in the offspring and then optimised during the fitness evaluation. The whole process presenting the Genetic Programming (GP) methodology in this paper is shown schematically in Figure 6.

More details and implementations about genetic programming used in this paper can be found in Armani (2014). 


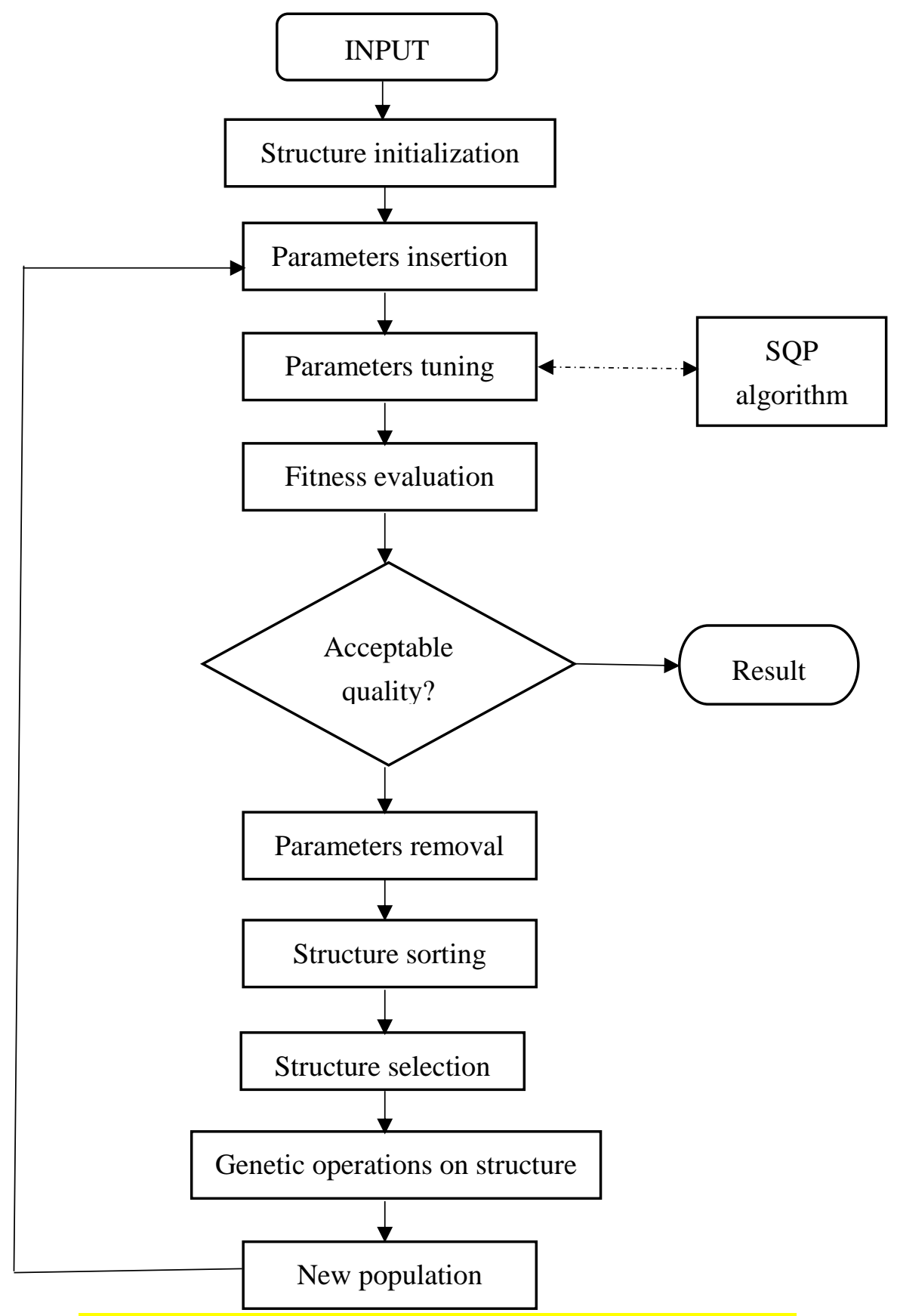

Figure 6. A flowchart of Genetic Programming methodology. 
In order to encourage the evolution of compact, smooth, and accurate mathematical expressions and to avoid 'bloating' (Poli et al. 2008), the definition of fitness values $F(i, t)$ of individual $i$ at generation $t$ has been represented in the form of a weighted sum of different terms or objectives, as an approach to solve the multi-objective optimization using the evolution-based algorithm:

$$
\begin{aligned}
& F(i, t)=a_{1} F_{1}(i, t)+a_{2} F_{2}(i, t)+a_{3} 10^{6} F_{3}(i, t)+a_{4} F_{4}(i, t) \\
& a_{1}+a_{2}+a_{3}+a_{4}=1
\end{aligned}
$$

where $F_{1}$ is the root mean square error (RMSE) of the $i$ th individual in the $t$ th generation evaluated on the given data set, divided by the average RMSE of the archive individuals at the previous generation; $F_{2}$ is the square of the number of numerical coefficients (parameters) present in the individual; $F_{3}$ is the number of operations not defined (i.e. division by zero) in the individual at any of the DOE sample point; $F_{4}$ is the number of nodes that the individual is made of and $\mathrm{a}_{1}, \mathrm{a}_{2}$, $\mathrm{a}_{3}$ and $\mathrm{a}_{4}$ are weighting factors (that add up to 1 ) determined by the exhaustive testing and tuning of the GP algorithm (Armami et al. 2011). Their values were: $\mathrm{a}_{1}=0.8989, \mathrm{a}_{2}=0.001, \mathrm{a}_{3}=0.1$ and $\mathrm{a}_{4}=0.0001$.

In total, $140 \mathrm{FE}$ analyses of the PFT device (corresponding to $140 \mathrm{DoE}$ points) are performed to generate the response sets for building the metamodels, which will be used to explore the 
structural properties of different geometries for the maximal output power of the PFT device in the optimization section.

\section{Finite element analysis of Piezoelectric flex transducer (PFT)}

Using the ANSYS Parametric Design Language (APDL), a finite element analysis tool for the parameterized modelling of the PFT is developed as a large number of similar models must be analyzed to study the responses of the PFT for the purpose of design optimization. The correctness of the CPC-FEM model has been described in previous section. This automated tool is able to generate efficient and reliable models, facilitate data collection, as well as alter the PFT geometry with acceptable programming effort in order to analyse their mechanical-electrical coupled responses under specified loads. Detailed information about the FE modelling of the PFT has been given in the Development of the coupled piezoelectric-circuit finite element model and its validation Section, and the material properties of the device are listed in Table 1. A mesh sensitivity study is also performed to obtain a converged solution, as shown in the Convergence analysis of the developed PFT Section.

Table 1. Material properties used in PFT. 


\begin{tabular}{|c|c|}
\hline \multicolumn{2}{|c|}{ AK Stainless Steel: Austenitic stainless steel 304, MatWeb, LLC } \\
\hline Young's Modulus (GPa) & 193 \\
\hline Yield Strength (MPa) & 251 \\
\hline Density $\left(\mathrm{Kg} / \mathrm{m}^{\wedge} 3\right)$ & 8030 \\
\hline Poisson's Ratio & 0.24 \\
\hline \multicolumn{2}{|c|}{ Piezoelectric Material: DeL Piezo DL-53HD } \\
\hline \multicolumn{2}{|l|}{ Elastic Compliance $\left(\mathrm{x} 10^{\wedge}-12 \mathrm{~m}^{\wedge} 3 / \mathrm{N}\right)$} \\
\hline $\mathrm{S}_{11}$ & 15.1 \\
\hline $\mathrm{S}_{12}$ & -4.5 \\
\hline $\mathrm{S}_{13}$ & -9.4 \\
\hline $\mathrm{S}_{33}$ & 24.8 \\
\hline $\mathrm{S}_{44}$ & 37.1 \\
\hline $\mathrm{S}_{66}$ & 39.2 \\
\hline \multicolumn{2}{|c|}{ Relative Dielectric Constant (at the constant stress) } \\
\hline$\varepsilon^{\mathrm{T}}{ }_{\mathrm{r} 11}$ & 3550 \\
\hline$\varepsilon^{\mathrm{T}} \mathrm{r} 33$ & 3850 \\
\hline Density $\left(\mathrm{Kg} / \mathrm{m}^{\wedge} 3\right)$ & 7900 \\
\hline \multicolumn{2}{|c|}{ Piezoelectric strain constants $\left(\mathrm{x} 10^{\wedge}-12 \mathrm{~m} /\right.$ volt $)$} \\
\hline$d_{15}$ & 810 \\
\hline $\mathrm{d}_{31}$ & -300 \\
\hline$d_{33}$ & 680 \\
\hline Piezoelectric Coupling Coefficient $\mathrm{k}_{31}$ & 0.42 \\
\hline Mechanical quality factor $Q$ & 20 \\
\hline
\end{tabular}


Convergence analysis of the developed PFT

The boundary conditions of the simulations for the PFT are shown in Figure 7. The fix base is set at the bottom endcap while the distribute load applies at the top endcap. The magnitude of the load is $1 \mathrm{kN}$. The resistor is connected between the top and bottom surface of the piezoelectric layer.

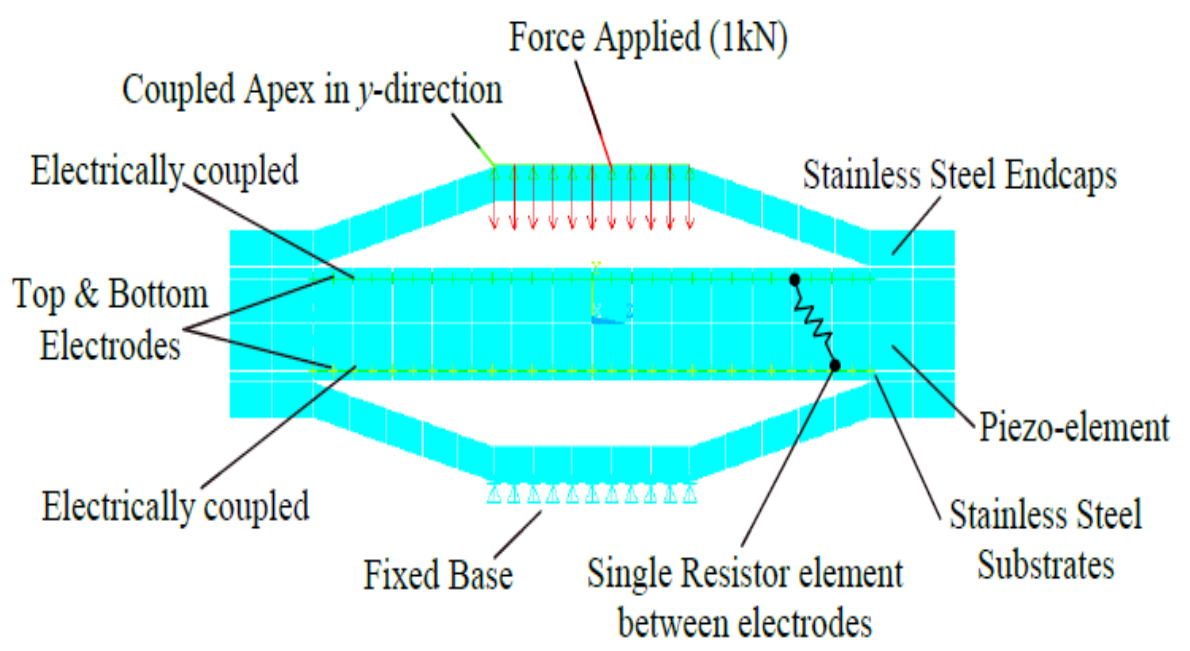

Figure 7. Boundary conditions of PFT simulations.

In theory, more accurate FE solution of a mathematical model can always been achieved with finer meshes. However, increasing the accuracy of the FE model by reducing the mesh size will be penalised by significant increase of computing time. Therefore, before building the metamodel 
using GP, a mesh convergence study needs to be performed to identify an appropriate mesh size for achieving sufficient accuracy of the FE analysis with acceptable computational cost. The initial CPC-FEM model for the PFT had an element size of $3 \mathrm{~mm}$ in length, height and width. In order to find a more favourable element size, the electric power output and the maximal principal stress are plotted against measure of mesh density in Figures 7 and 8 . The numbers along the $\mathrm{x}$ axis in the Figures stand for the integer that are used to divide the original mesh size, for example, 4 stands for $1 / 4$ of the original size.

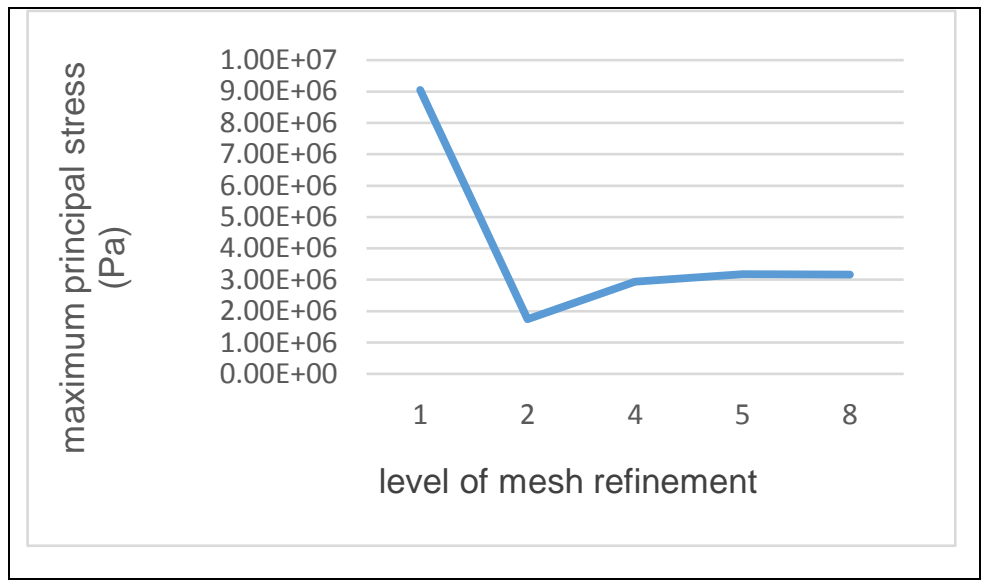

Figure 8. Maximum principal stress vs. mesh size. 


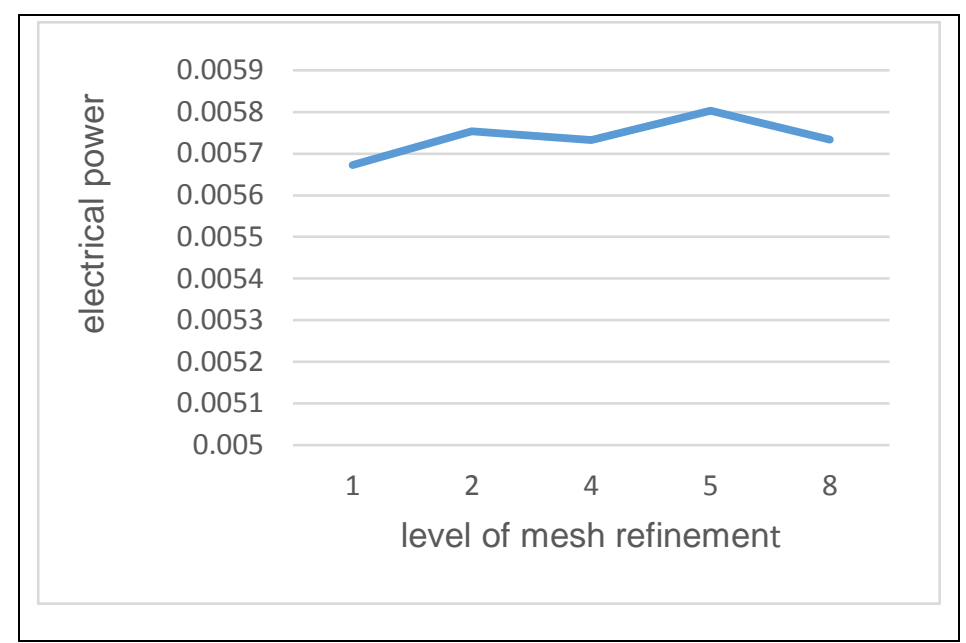

Figure 9. Electrical power vs. mesh size.

From Figures 8 and 9, it is clear that the predicted electric power converges faster than the maximum principal stress does before the level of mesh refinement is below 4 . The slower convergence of the principal stress was due to the fact that the stress was calculated point by point within the materials, while the electric outputs were calculated from the average stress of the material, which are less sensitive to mesh size.

By changing the size of the element required in the PFT model, the variations of magnitudes of the output current $(\mathrm{I})$, voltage $(\mathrm{V})$ and power $(\mathrm{P})$ are shown in Table 2. It is obvious that a good convergence has been achieved for all the electric outputs when the mesh size is reduced to four times less than the original size. 
Table 2. Electrical output vs. mesh size.

\begin{tabular}{|l|l|l|l|}
\hline & I & V & P \\
\hline original mesh & $-2.49739 \mathrm{E}-05$ & -249.739 & 0.00567255 \\
\hline mesh/2 & $-2.51175 \mathrm{E}-05$ & -251.175 & 0.00575408 \\
\hline mesh/4 & $-2.50425 \mathrm{E}-05$ & -250.425 & 0.00573282 \\
\hline mesh/5 & $-2.52266 \mathrm{E}-05$ & -252.266 & 0.00580307 \\
\hline mesh/8 & $-2.50340 \mathrm{E}-05$ & -250.34 & 0.00573358 \\
\hline
\end{tabular}

Based on the convergence analysis shown in Figures 8, 9 and Table 2, it can be concluded that the FE results of the PFT model are sufficiently accurate when the element size is reduced to $1 / 4$ of the original element size, which is $0.75 \mathrm{~mm}$.

\section{Design Variables, Constraints and Loads for Parametric Optimization}

The PFT device consists of a piezoelectric (PZT) plate sandwiched between two metal endcaps with shallow cavities as shown in Figure 10 and its CAD sketch is depicted in Figure 11. Two geometric parameters, i.e., the total length $(\mathrm{D}=52 \mathrm{~mm})$ and the width $(\mathrm{W}=30 \mathrm{~mm})$, are kept constant in the optimization process. Clearly, the effective design should amplify and redirect the downward applied mechanical force to a horizontal force so that the majority of the applied force acts to stretch piezo-plate horizontally. The design should also allow a certain amount of space 
between the piezo-plate and the endcaps so that the piezo-plate can actuate more freely for a higher elastic deformation or elastic strain, which will generate more electricity.

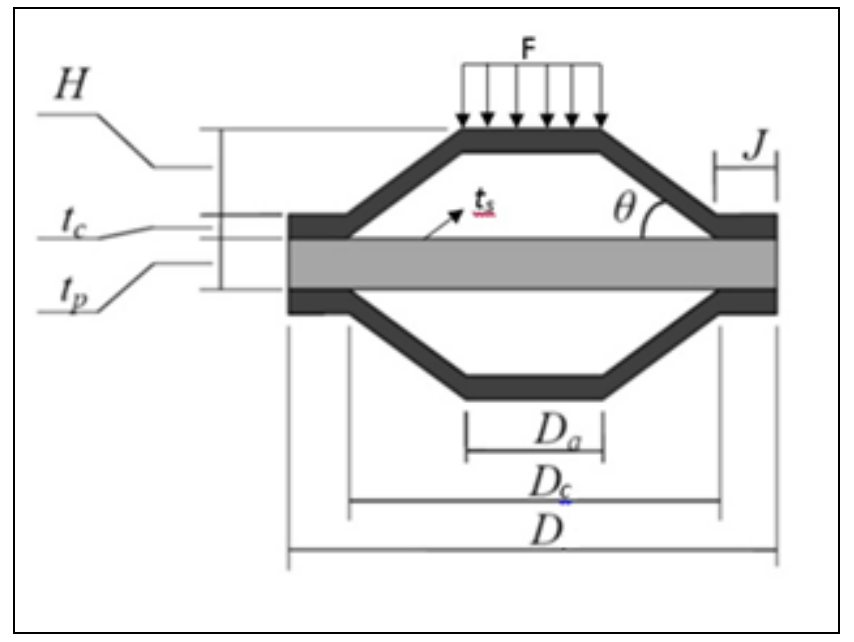

Figure 10. Geometrical parameters describing the PFT configuration.

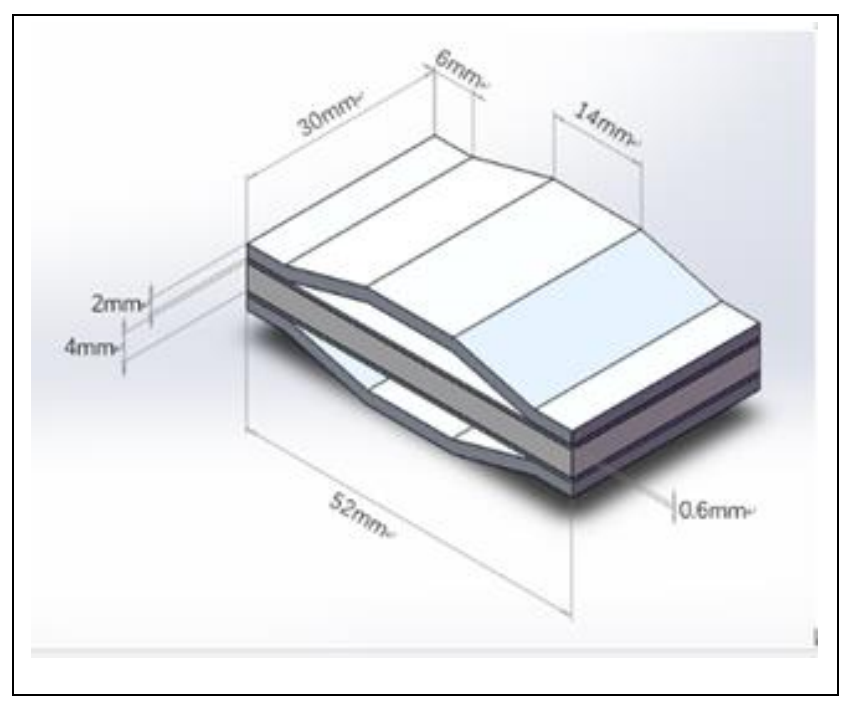

Figure 11. CAD sketch of three-dimensional PFT geometry. 
Taking into account all the above, the design variables chosen to vary the geometry of the PTF include the cavity length $(D c)$, the apex length $\left(D_{a}\right)$, the endcap internal angle $(\theta)$ and the respective thicknesses of the piezoelectric plate $\left(t_{p}\right)$, the substrate layer $\left(t_{s}\right)$, and the cap $\left(t_{c}\right)$. By altering the cavity length, the internal angle, and the apex length, the overall height of the cap $(H)$ will be changed. The joint length $(J)$ can be defined as a function of the cavity length. Consequently, seven parameters are selected as the design variables in the optimization process of the PTF for maximum power generation. The design parameters are summarized in Table 3 with their minimum and maximum bounds given for the practical and manufacturing restraints. Other constraints are also considered in the optimization. The measure of the maximum principal stress has been taken throughout the structure and the critical areas have been identified nearby the sharp corners of the endcap component. Hence, the endcap fails if it is stressed beyond the strength of the material. In this research, a safety factor of 2.0 has been selected for the strength constraint that will be activated when the maximum principal stress reaches the half value of the yield stress of the endcap material. The logic behind this is to find a desirable and practical design, which is in the feasible region far away from the brink of violating the yield stress constraint. The apex downward displacement should not exceed the height $H$ so that the contact between the cap and the substrate can be avoided in the process of energy harvesting. Thus, the optimization problem is formulated as shown below: 
$\operatorname{Max} P$

Subject to:

$$
\begin{aligned}
& \frac{D_{\text {disp }}}{H}<1 \\
& \frac{\sigma}{\sigma_{y}} \leq 0.5
\end{aligned}
$$

where $P$ means the non-dimensional electrical power and it is normalized by the maximum electrical power among all 140 sampling points without considering yielding of the material. This rule is also employed to calculate the normalized stress and displacement. The principal stress is normalized by the yield stress of the material and the displacement of the metal cap is normalized by the distance between the metal cap and the piezoelectric material.

$H$ is the height of endcap and can be computed by

$$
H=\frac{D_{c}-D_{a}}{2} \tan \theta
$$

$J$ is the joint length,

$$
J=\frac{D_{p}-D_{c}}{2}
$$

$D_{\text {disp }}$ is the displacement of the apex, 
$\sigma_{y}$ is the yield stress of the endcap material,

$\sigma$ is the maximum principal stress in the cap component.

Table 3. Bounds of Design variables

\begin{tabular}{|l|l|l|}
\hline DVs & Lower Bound & Upper Bound \\
\hline Thickness of PZT, $\mathrm{t}_{\mathrm{p}}(\mathrm{mm})$ & 0.5 & 9 \\
\hline Thickness of substrate, $\mathrm{t}_{\mathrm{s}}(\mathrm{mm})$ & 0.6 & 0.9 \\
\hline Thickness of endcap, $\mathrm{t}_{\mathrm{c}}(\mathrm{mm})$ & 0.5 & 4 \\
\hline Length of cavity, $\mathrm{D}_{\mathrm{c}}(\mathrm{mm})$ & 25 & 40 \\
\hline Length of apex, $\mathrm{D}_{\mathrm{a}}(\mathrm{mm})$ & 9.8 & 18.2 \\
\hline Internal angle, $\theta(\mathrm{deg})$ & 5 & 45 \\
\hline Resistive loads, $\mathrm{R}(\mathrm{M} \Omega)$ & 1 & 19 \\
\hline
\end{tabular}

A uniformly distributed downward pressure is applied to the upper surface of the upper endcap as shown in Figure 10. The total force applied to the top endcap is $1 \mathrm{kN}$ at a low frequency of $2 \mathrm{~Hz}$ and this is an approximation of what a solider might exert on the ground. 
The explicit expressions for the responses related to the electrical power, current, voltage, normalized maximum principal stress of the cap component, and displacement of the apex in the PFT design are built by GP which is described in the section Metamodel building by Genetic Programming (GP). As an example, the expression for the normalized maximum principal stress is:

$$
\begin{aligned}
& \sigma=0.2614-0.01449 \mathrm{Z} 3+0.002264 \mathrm{Z} 4-\frac{0.0027 \mathrm{Z} 5}{\mathrm{Z} 1}+\frac{1.92 \mathrm{Z} 5}{\mathrm{Z} 2 \mathrm{Z} 4^{2}}-\frac{9.429 \mathrm{Z} 5}{\mathrm{Z} 4 \mathrm{Z} 6}-0.0028 \mathrm{Z} 6 \\
& +\frac{0.002746\left(-2.71206 \mathrm{Z} 4-\frac{3.10105 \mathrm{ZZZ} 4}{\mathrm{Z} 2}+1.64421 \mathrm{Z6}\right)}{\mathrm{Z}^{2}}+\frac{0.00000634 \mathrm{Z} 6^{6}}{\mathrm{Z}^{6} \mathrm{Z3}^{6} \mathrm{Z}^{2} \mathrm{Z}^{3} \mathrm{Z} 7} \\
& +\frac{1}{\mathrm{Z} 6} 0.0463\left(86.2934-\frac{3.48637 \mathrm{Z} 1 \mathrm{Z3}^{2}}{\mathrm{Z}^{2} \mathrm{Z} 4}-2.14548 \mathrm{Z} 4+\frac{17.4637 \mathrm{Z} 5}{\mathrm{Z} 3}\right. \\
& -\frac{187.722 \mathrm{Z} 5}{\mathrm{Z} 3 \mathrm{Z} 4}-1 /(\mathrm{Z} 2 \mathrm{Z} 3 \mathrm{Z} 6) 1.891 \mathrm{Z} 4\left(17.7753+\frac{28.1429 \mathrm{Z} 5^{2}}{\mathrm{Z} 2 \mathrm{Z}^{2}}-\frac{113.415 \mathrm{Z} 5}{\mathrm{Z} 4 \mathrm{Z} 6}\right.
\end{aligned}
$$

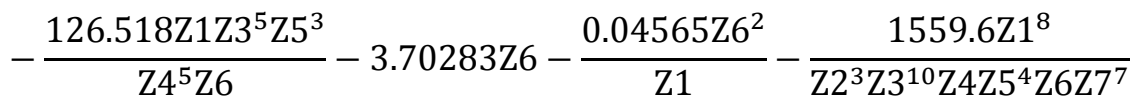

$$
\begin{aligned}
& -\frac{0.3615 \mathrm{Z} 6 \mathrm{Z7}}{\mathrm{Z} 3 \mathrm{Z} 4}
\end{aligned}
$$

where $Z 1$ to $Z 7$ are the design variables detailed in Table 4. A graphical representation of the quality of the fit of the GP approximation for the normalized maximum principal stress $\sigma$ is shown in Figure 12, where a point on the diagonal represents a perfect fit. 


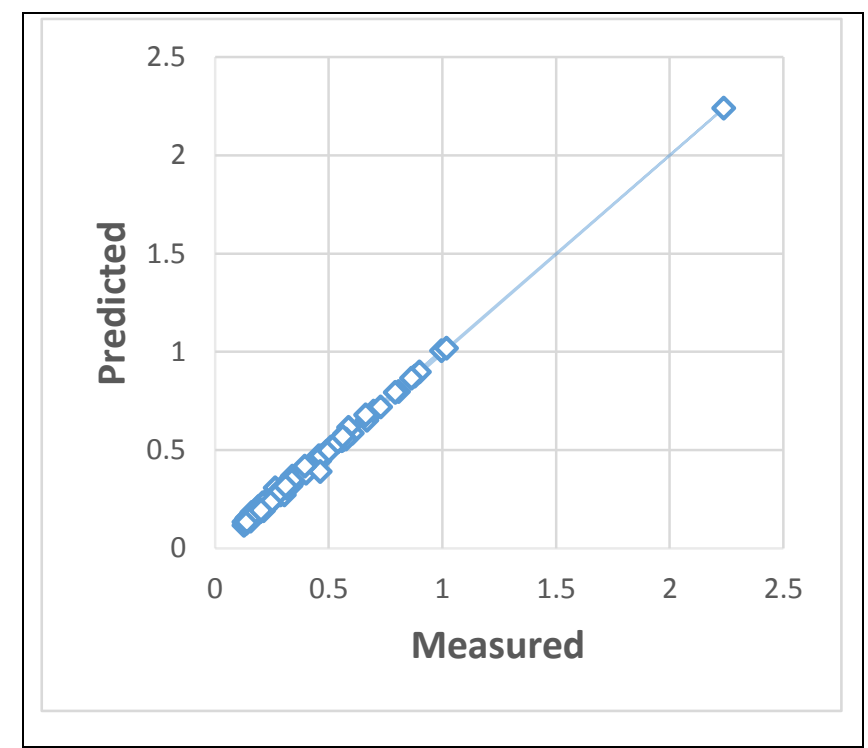

Figure 12. Indications of the difference between the normalized maximum principal stress response (predicted) and the training data (measured)

In this paper, the parametric optimization of the GP-derived analytical metamodels for the novel PFT device was performed by Sequential Quadratic Programming (SQP) technique. SQP (Wilson, 1963 and Powell, 1977) is widely used to solve nonlinear constrained optimization problems due to its typically fast convergence. With a solid theoretical and computational foundation, SQP algorithms have been developed and used to solve a remarkably large set of practical problems with continuous design variables. Since SQP is sensitive to the selection of initial designs, optimizations with three different initial designs, shown in Table 4 as Case one, Case two and Case three, respectively, are performed to obtain a converged optimal solution. Numerical results 
shown that the predicted optimal power outputs and the associated maximum principle stresses from the three initial designs converged to almost the same optimal solution. Without loss of generality, the predicted optimal power output of design Case one using the metamodel-based optimization technique (see Table 4) is compared with the one before optimization and the one from FE validation on the optimized design (see Table 5).

Table 4. Optimal designs of the PFT using SQP with different starting points

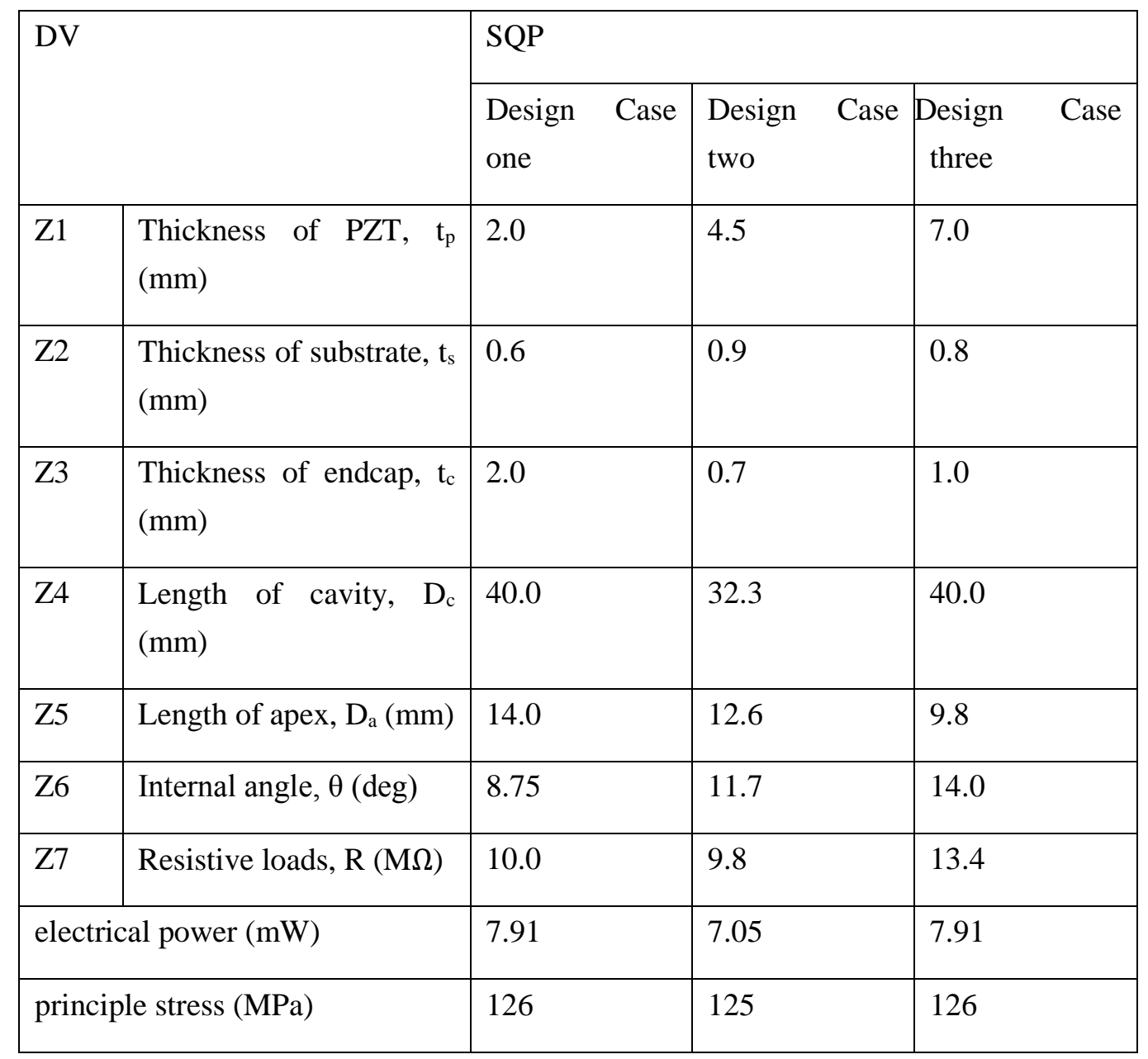


Table 5. Structural and electrical response values for the optimum design case one.

\begin{tabular}{|l|l|l|l|}
\hline Structural response type & $\begin{array}{l}\text { Normalized } \\
\text { Electrical power }\end{array}$ & $\begin{array}{l}\text { Normalized maximum } \\
\text { principal stress }\end{array}$ & $\begin{array}{l}\text { Normalized } \\
\text { displacement }\end{array}$ \\
\hline $\begin{array}{l}\text { Results before optimization } \\
\text { (Daniels, 2014) }\end{array}$ & 0.24 & 0.51 & 0.01 \\
\hline Predicted by metamodel & 0.46 & 0.50 & 0.01 \\
\hline Validation by FE analysis & 0.38 & 0.49 & 0.009 \\
\hline
\end{tabular}

The initial design Case one in Table 4 is the design of Daniels (2014) before optimization giving a normalized electric power $4.13 \mathrm{~mW}$ and a maximum stress of $128 \mathrm{MPa}$. After optimization using the metamodel, the electric power is increased to $7.91 \mathrm{~mW}$, though a detailed FE analysis on the optimized design produces a power of $6.54 \mathrm{~mW}$, representing an improvement of more than $58 \%$.

The results in Table 5 show also that the output power is very sensitive to the structural stress in the device, especially in searching the optimal design. Considering the PFT design with a safety factor of 2.0, the metamodel-predicted optimum has a zero margin of principal stress of 0.50 with a normalized electrical power of 0.46 . However, the normalized electrical power computed from the FE model for the optimized design is smaller (0.38), representing a total of $6.5 \mathrm{~mW}$ harvested from the device, with a small increase of the stress margin by 0.01 . The discrepancies between the metamodel and the FE results can be explained by the limited sampling points (140 points) in 
the design space for generating a globally accurate surrogate. To address this issue, a global-local metamodel strategy will be proposed to predict the response of electrical power more accurately in future research. The normalized displacement $(0.01)$ predicted by the metamodel is slightly larger than the displacement (0.009) obtained by the FE analysis. This is acceptable as it is not the critical constraint in this design optimization problem. In comparison with the results from the original design (Daniels, 2014), the normalized electrical power generated by the optimal design is increased from 0.24 to 0.38 (by 58\%) and the value of the normalized maximum principal stress $(0.49)$ is very close to half of the stress limit defined in the stress constraint. For a better comparison, the design variables of the original and the final optimum designs are listed in Table 6.

Table 6. Optimum Design variables subjected to the stress and displacement constraints.

\begin{tabular}{|l|l|l|l|l|l|l|l|}
\hline $\begin{array}{l}\text { Design } \\
\text { variable } \\
\mathrm{s}\end{array}$ & $\begin{array}{l}\text { Thickness } \\
\text { of PZT, } \\
\mathrm{t}_{\mathrm{p}}(\mathrm{mm})\end{array}$ & $\begin{array}{l}\text { Thickness } \\
\text { of } \\
\text { substrate, } \\
(\mathrm{mm})\end{array}$ & $\begin{array}{l}\text { Thickness } \\
\text { of } \\
\text { endcap, } \mathrm{t}_{\mathrm{c}} \\
(\mathrm{mm})\end{array}$ & $\begin{array}{l}\text { Length of } \\
\text { cavity, } \\
\mathrm{D}_{\mathrm{c}}(\mathrm{mm})\end{array}$ & $\begin{array}{l}\text { Length } \\
\text { of apex, } \\
\mathrm{D}_{\mathrm{a}}(\mathrm{mm})\end{array}$ & $\begin{array}{l}\text { Internal } \\
\text { angle, } \theta \\
(\mathrm{deg})\end{array}$ & $\begin{array}{l}\text { Resistiv } \\
\mathrm{e} \text { loads, } \\
\mathrm{R}(\mathrm{M} \Omega)\end{array}$ \\
\hline $\begin{array}{l}\text { Original } \\
\text { value }\end{array}$ & 4 & 0.6 & 2 & 40 & 14 & 15.07 & 10 \\
\hline $\begin{array}{l}\text { Optimum } \\
\text { value }\end{array}$ & 9 & 0.6 & 1.8 & 40 & 10 & 16 & 18.5 \\
\hline
\end{tabular}


The thickness of the optimal PFT design is $9 \mathrm{~mm}$ reaching its upper bound. The same observation applies to some other design variables, such as the length of cavity and the resistive loads. However, the optimal values for the thickness of the substrate and the apex length are very close to the lower bounds. As expected, the thickness of the endcap and the internal angle are the key parameters that significantly affect the electrical power generation of the PFT. The smaller they are, the higher the electrical power output will be.

\section{Conclusions}

A metamodel-assisted optimization approach for designing piezoelectric flex transducer (PFT) was proposed to generate the maximum electrical power output from the biokinetic energy of human body movement. The PFT was modelled by coupled piezoelectric-circuit finite element and further optimized using the Sequentail Quadratic Programming technique on the metamodels generated with Genetic Programming from a 140-point optimal Latin hypercube design of experiments. The optimized design was further validated by finite element simulations. When a safety design factor of 2.0 is applied, the magnitude of the electrical power generated from the optimal PFT harvesting device was up to $6.5 \mathrm{mw}$, representing a more than $58 \%$ improvement of the initial design. It is concluded that the global metamodel-assisted optimization approach allows 
the problem to be solved with acceptable accuracy and provides the designers with a wealth of information on the structural behaviour and energy output of the PFT harvesting device.

\section{Reference}

ANSYS, Software Package, Ver. 13, ANSYS, Inc., Canonsburg, PA, 2010.

Anton SR, Sodano HA (2006) A review of power harvesting using piezoelectric materials (20032006). Smart Materials and Structures 16 (2007) R1-R21.

Audze P, Eglais V (1977) New approach for planning out of experiments. Problems of Dynamics and Strengths 35:104-107, Zinatne Publishing House, Riga.

Armani U, Khatir Z, Khan A, Toropov VV, Polyinkin A, Thompson H, Kapur N, Noakes CJ (2011) Control of physical consistency in meta-model building by genetic programming. In: Proceedings of the Second International Conference on Soft Computing Technology in Civil, Structural and Environmental Engineering, Civil-Comp Press, Stirlingshire, UK, Paper 43, 2011. doi:10.4203/ccp.97.43.

Armani U (2014) Development of a hybrid genetic programming technique for computationally expensive optimisation problems. Dissertation, University of Leeds. 
Box JF (1980) R. A. Fisher and the Design of Experiments, 1922-1926, The American Statistician, Vol. 34, No. 1, pp. 1-7.

Daniels A (2014) Design, analysis and fabrication of a mobile energy harvesting device to scavenge bio-kinetic energy. Dissertation. Cranfield university.

Daniels A, Zhu M, Tiwari A (2013) Design, analysis and testing of a piezoelectric flex transducer for harvesting bio-kinetic energy. Journal of Physics: Conference Series 476, 012047.

Deng L, Wen Q, Jiang S, Zhao X and She Y (2015) On the optimization of piezoelectric vibration energy harvester. Journal of Intelligent Material Systems and Structures 2015, Vol. 26(18): 2489-2499.

Forrester AIJ, Sóbester A, Keane AJ (2008) Engineering Design via Surrogate Modelling - A Practical Guide. World Health, 2008.

Harb A (2011) Energy harvesting: State-of-the-art. Renewable Energy 36 (2011): 2641-2654.

Khalid A, Redhewal AK, Kumar M and Srivastav A (2015) Piezoelectric Vibration Harvesters Based on Vibrations of Cantilevered Bimorphs: A Review. Materials Sciences and Applications, 2015(6): 818-827. 
Kiyono, CY Silva ECN and Reddy JN (2016) Optimal design of laminated piezocomposite energy harvesting devices considering stress constraints. International journal for numerical methods in engineering 2016(105):883-914

Kim HS, Kim JH and Kim J (2011) A review of piezoelectric energy harvesting based on vibration. International journal of precision engineering and manufacturing Vol. 12, No. 6, pp. 1129-1141

Kim H, Priya S, Stephanou H and Uchino K (2007) Consideration of impedance matching techniques for efficient piezoelectric energy harvesting, IEEE transactions on ultrasonics, ferroelectrics, and frequency control. 54 (2007): 1851-1858.

Krishna T. Settaluri, Hsinyi L and Rajeev JR (2011) Thin thermoelectric generator system for body energy harvesting. Journal of Electronic Materials, Vol. 41, No. 6, 2012.

Krishna VS, Mohamed SMA (2014) Micro-scale energy harvesting devices: review of methodological performances in the last decade. Renewable and Sustainable Energy Reviews 54 (2016): 1035-1047.

Kögl M, Silva ECN (2005) Topology optimization of smart structures: design of piezoelectric plate and shell actuators. Smart Materials and Structures 14(2):387-399 
Lu C, Tsui C, and Ki W (2011) Vibration energy scavenging system with maximum power tracking for micropower applications. IEEE transactions on very large scale integration (vlsi) systems, vol. 19 , no. 11 .

Li H, Tian C, and Deng ZD (2014) Energy harvesting from low frequency applications using piezoelectric materials. Applied physics reviews 1, 041301.

Newnham RE, Zhang J and Meyer JRR (2000) Cymbal transducers: a review. IEEE Applications of Ferroelectrics, 2000 Page(s): 29 - 32 vol. 1.

Reinhard L (1990) Simulation of Piezoelectric Devices by Two- and Three-Dimensional Finite Elements. IEEE transactions on ultrasonics, ferroelectrics, and frequency control. 37 233-47.

Siddique ARM, Mahmud S, Heyst BV (2015) A comprehensive review on vibration based micro power generators using electromagnetic and piezoelectric transducer mechanisms. Energy Conversion and Management 106 (2015) 728-747.

Sohn, H, Farrar CR, Hemez F et al. (2002) A Review of Structural Health Monitoring Literature: 1996-2001. Masachusets, USA 2002.

Silva ECN and Kikuchi N (1999) Design of piezoelectric transducers using topology optimization. Smart Materials and Structures 8 (1999): 350-364. 
Toropov VV, Bates SJ, Querin OM (2007) Generation of extended uniform Latin hypercube designs of experiments. In: 9th International Conference on the Application of Artificial Intelligence to Civil, Structural and Environmental Engineering, St. Julians, Malta, Civil-Comp Press, Stirlingshire, UK, September, Paper 7, 2007. doi:10.4203/ccp.87.7

Koza JR (1992) Genetic Programming: On the Programming of Computers by Means of Natural Selection. MIT press, Cambridge, USA.

Olodort R and Cazalet P (2004) Portable communication devices. Google Patents, 2005, Available at: www.google.com/patents/US20050125570 (Accessed 3 June 2016).

Poli R, Langdon WB and McPhee NF (2008) A field guide to genetic programming. With contributions by J. R. Koza, Available at: www.gp-field-guide.org.uk. (Accessed 21 June 2016).

Powell MJD (1977) A Fast Algorithm for Nonlinearly Constrained Optimization Calculations. In: Numerical Analysis: Proceedings of the Biennial Conference Held at Dundee. Berlin, Heidelberg, pp. 144-157.

Wilson RB (1963) A Simplicial Method for Convex Programming. Dissertation. Harvard University. 
Zhao L, Tang L and Yang Y (2015) Synchronized charge extraction in galloping piezoelectric energy harvesting. Journal of Intelligent Material Systems and Structures. 2016, Vol. 27(4) 453-468.

Zhou S, Chen W, Mohammad HM, et al. (2016) Design and modeling of a flexible longitudinal zigzag structure for enhanced vibration energy harvesting. Journal of Intelligent Material Systems and Structures. Published online before print May 3, 2016, doi:10.1177/1045389X16645862 